\title{
Sox2 suppresses the invasiveness of breast cancer cells via a mechanism that is dependent on Twist 1 and the status of Sox 2 transcription activity
}

Fang $\mathrm{Wu}^{1 \dagger}$, Xiaoxia $\mathrm{Ye}^{1 \dagger}$, Peng Wang ${ }^{1}$, Karen Jung ${ }^{2}$, Chengsheng $\mathrm{Wu}^{1}$, Donna Douglas ${ }^{3}$, Norman Kneteman ${ }^{3}$, Gilbert Bigras ${ }^{1}$, Yupo $\mathrm{Ma}^{4}$ and Raymond Lai ${ }^{1,2,5^{*}}$

\begin{abstract}
Background: Sox2, an embryonic stem cell marker, is aberrantly expressed in a subset of breast cancer (BC). While the aberrant expression of Sox2 has been shown to significantly correlate with a number of clinicopathologic parameters in $\mathrm{BC}$, its biological significance in $\mathrm{BC}$ is incompletely understood.

Methods: In-vitro invasion assay was used to evaluate whether the expression of Sox 2 is linked to the invasiveness of MCF7 and ZR751 cells. Quantitative reverse transcriptase-polymerase chain reaction (qRT-PCR) and/or Western blots were used to assess if Sox2 modulates the expression of factors known to regulate epithelial mesenchymal transition (EMT), such as Twist1. Chromatin immunoprecipitation (ChIP) was used to assess the binding of Sox2 to the promoter region of Twist1.

Results: We found that siRNA knockdown of Sox2 expression significantly increased the invasiveness of MCF7 and ZR751 cells. However, when MCF7 cells were separated into two distinct subsets based on their differential responsiveness to the Sox2 reporter, the Sox2-mediated effects on invasiveness was observed only in 'reporter un-responsive' cells (RU cells) but not 'reporter responsive' cells (RR cells). Correlating with these findings, siRNA knockdown of Sox2 in RU cells, but not RR cells, dramatically increased the expression of Twist1. Accordingly, using ChIP, we found evidence that Sox2 binds to the promoter region of Twist1 in RU cells only. Lastly, siRNA knockdown of Twist1 largely abrogated the regulatory effect of Sox 2 on the invasiveness in RU cells, suggesting that the observed Sox2-mediated effects are Twist1-dependent.

Conclusion: Sox2 regulates the invasiveness of BC cells via a mechanism that is dependent on Twist1 and the transcriptional status of Sox2. Our results have further highlighted a new level of biological complexity and heterogeneity of BC cells that may carry significant clinical implications.
\end{abstract}

Keywords: Sox2, Transcription activity, Invasiveness, Twist1, Breast cancer

\footnotetext{
* Correspondence: rlai@ualberta.ca

${ }^{\dagger}$ Equal contributors

'Department of Laboratory Medicine and Pathology, University of Alberta, Edmonton, Alberta, Canada

${ }^{2}$ Department of Oncology, University of Alberta, Edmonton, Alberta, Canada

Full list of author information is available at the end of the article
} 


\section{Background}

Tumor invasiveness is a complex process in which malignant cells dissociate and migrate from the primary site of growth, which may eventually lead to the formation of distant metastases [1]. In many types of solid tumor, it has been shown that epithelial-mesenchymal transition (EMT) is a crucial step for tumor invasiveness [2,3]. During EMT, malignant epithelial cells shed their differentiated characteristics (e.g. cell-cell adhesion, apical-basal polarity and immobility) and acquire mesenchymal features (e.g. increased motility and invasiveness) [4]. The induction of EMT can be triggered by cytokines, such as TGF- $\beta$ and interleukin (IL)-8, as well as several transcriptional factors including Twist1, Snail, and ZEB [5-9]. Twist1 has been described to be one of the key promoters of EMT and invasiveness in a number of cancer types [10-12]. In several studies, Twist1 was found to be up-regulated by a number of proteins including STAT3 [13], BMP2 [14], SRC-1 [15], MSX2 [16], NF-kB [17], and ILK [18] and down-regulated by miR-580 and CPEB1/2 [19]. In breast cancer (BC), Twist1 has been found to promote EMT and invasiveness [5]. A number of immunohistochemical studies have described a significant positive correlation between Twist1 and the metastatic/invasive property of $\mathrm{BC}$ [5-8]. In an animal model, siRNA knockdown of Twist1 was found to inhibit BC cells to metastasize to the lungs [5]. Furthermore, the mechanisms by which Twist1 promotes tumor invasiveness in $\mathrm{BC}$ have been extensively examined; down-regulation of E-cadherin [9] and up-regulation of SET8 [20], AKT2 [8], miRNA-10b [21], IL8 [22] and PDGF $\alpha$ [23] have been implicated.

Sox2 (sex determining region Y-box protein 2) is a transcription factor that plays a key role in maintaining the pluripotency of embryonic stem cells [24-26]. The importance of Sox 2 in stem cell biology is highlighted by the fact that Sox 2 represents one of the 4 genes implicated in the conversion of fibroblasts into inducible pluripotent stem cells [27,28]. Recent studies have shown that Sox2 is aberrantly expressed in several types of solid tumors, including BC, lung cancer, prostate cancer, glioblastomas and melanomas [29-33]. The expression of Sox 2 detectable by immunohistochemistry has been found to positively correlate with the invasiveness and metastatic potential of several types of solid tumors [34-37]. Nevertheless, in-vitro studies that directly assess the role of Sox2 in regulating tumor invasiveness are relatively scarce [35-38]. In several types of cancer cells (e.g., gliomas, melanomas and colorectal cancer), knockdown of Sox 2 using siRNA was found to decrease invasiveness [35-37]. In one study, enforced expression of Sox2 in MCF7, an estrogen receptor-positive (ER+) BC cell line, was found to increase invasiveness in an invitro assay by approximately 60\% [38]. The mechanisms by which Sox 2 regulates the invasiveness of $\mathrm{BC}$ cells are largely unknown. For instance, whether the regulatory effects of Sox2 on the invasiveness of $\mathrm{BC}$ are linked to regulators of EMT (such as Twist1) has not been examined previously.

In this study, we aimed to further define the roles of Sox 2 in regulating the invasiveness of $\mathrm{BC}$ cells. In contradiction with the conclusion of a recently published paper [38], we found that Sox2 suppresses, rather than increases, the invasiveness of MCF7 cells. Furthermore, this biological effect is dependent on the regulation of Twist1 expression by Sox 2 . When we assessed the roles of Sox2 in the two distinct cell subsets of MCF7 separated based on their differential responsiveness to the Sox2 reporter, as shown previously [39], we found that the Sox2-mediated effects on invasiveness in $\mathrm{BC}$ is restricted to 'reporter un-responsive' (RU) cells. We believe that our results have shed important insights into the biological significance of Sox 2 in $\mathrm{BC}$, the invasiveness property of $\mathrm{BC}$, as well as a new level of biological complexity of this type of cancer.

\section{Methods}

\section{Cell culture}

MCF7 and ZR751 were purchased from American Type Culture Collection (ATCC, Rockville, MD). Both ZR751 and MCF7 cells were maintained in high glucose Dulbecco's Modified Eagle Medium (DMEM) (Life Technologies, Grand Island, NY) supplemented with $10 \%$ fetal bovine serum (FBS) (Sigma, Oakville, ON, Canada) and were cultured under an atmosphere of $5 \% \mathrm{CO}_{2}$ at $37^{\circ} \mathrm{C}$.

\section{Generation of stable cell lines}

Stable cells expressing the Sox2 GFP reporter were generated as previously described [39]. Cells stably expressing the Sox2 GFP reporter were cultured in DMEM, supplemented with $10 \% \mathrm{FBS}, 100 \mathrm{U} / \mathrm{ml}$ penicillin, 100 $\mathrm{ng} / \mathrm{ml}$ streptomycin. $1 \mu \mathrm{g} / \mathrm{ml}$ of puromycin was added to the culture medium at all times. The generated stable cell clones were analyzed for GFP expression by flow cytometry every two weeks over a 4-month period. RR and RU cells were sorted out based on GFP expression and cultured separately. The two populations remained 98\% pure over 4 months.

\section{Gene silencing}

MCF7 and ZR751 cells were transfected with $1 \mathrm{nmol}$ of SMARTpool siRNA designed against Sox2 (Thermo Scientific). Scramble non-targeting siRNA (Thermo Scientific) was used as the negative control. For all siRNA transfection, a BTX 830 electroporation instrument (Harvard Apparatus, Holliston, MA) was used. For double knockdown experiments, SMARTpool siRNA designed against Twist1 from Thermo Scientific was used. 
Enforced expression of Sox2 in MCF7 cells was performed as previously described [39]. Briefly, pheonix packaging cells were transfected with either pMXs Sox 2 retroviral vector (Addgene, MA, USA) or empty vector according to the manufacturer's suggestion. MCF7 cells were infected with retroviral particles three times in 24 hour intervals. 48 hours after the final infection, cells were overnight starved and were then used to perform invasion assay.

\section{Western blotting}

Western blot analyses were performed as previously described [40,41]. The following antibodies were used: Sox2 (Cell Signaling Technologies), Twist1 (Santa Cruz), $\gamma$-Tubulin (Sigma).

\section{Cell viability}

Cell viability was determined using the 3-(4,5-dimethylthiazol-2-yl)-5-(3-carboxymethoxyphenyl)-2-(4sulfophenyl)-2H-tetrazolium, inner salt (MTS) assay (Promega, Madison, WI) according to the manufacturer's protocol.

\section{Cell invasion assay}

As previously described, we assessed cell invasiveness using the Cytoselect ${ }^{\mathrm{Tm}}$ 24-well cell invasion assay kit (Cell Biolabs, San Diego, CA, USA) according to the manufacture's protocol [42]. Briefly, cells were overnight starved prior to invasion assay. Approximately $1 \times 10^{5}$ cells in serum free medium were plated in the top chamber and medium supplemented with 10\% FBS was used as a chemo-attractant in the lower chamber. The cells were then allowed to invade the reconstituted basement membrane matrix for 24 hours. The invasive cells passed the membrane were then dissociated from membrane, lysed and quantified using CyQuant GR fluorescent Dye.

\section{Quantitative RT-PCR}

Total RNA was extracted using TRIzol according to the manufacturer's protocol. Quantitative RT-PCR was performed using Applied Biosystem Prism 7900HT instruments. The TaqMan gene expression assay (Applied Biosystems) used were: Hs01548727_m1 (MMP2), Hs00 234579_m1 (MMP9), Hs01675818_s1 (Twist1), Hs0102 3894_m1 (E-cadherin), Hs00362037_m1 (N-cadherin, Hs00232783_m1(ZEB1) and Hs00998133_m1 (TGF- $\beta)$. Primer sequences for Snail are: Forward 5'-acaaaggctg acagactcactg-3', Reward 5'-tgacagccattactcacagtcc-3'. Primer sequences for Slug are: Forward 5' -gtctctcctgcac aaacatgag-3', Reverse 5'-atgctcttgcagctctctctct-3'. Primer sequences for MMP3: Forward $5^{\prime}$-cactcacagacc tgactcggtt-3', Reverse 5'- aagcaggatcacagttggctgg-3'. Primer sequence for $F A K$ are Forward $5^{\prime}$-gccttatgacg aaatgctgggc-3', Reverse $5^{\prime}$ - cctgtcttctggactccatcct -3 '.
Human GAPDH was used as control. Expression of each gene was measured in triplicate.

\section{Chromatin immunoprecipitation (ChIP) assay}

ChIP assay was performed as our previously described [39]. The chromatin was extracted from MCF7-RR and RU cells. A normal rabbit IgG antibody and anti-Sox2 antibody (Santa Cruz) was then incubated with the chromatin. Isolated DNA was then amplified with Twist1 primers $(-1478$ to -1322 of transcriptional start site, 156 bp amplicons): Forward 5'-ggcgagtccgtactgagaag-3' Reverse $5^{\prime}$ - cgtttcaggtccatccctta-3'.

\section{Statistical analysis}

All the statistical analyses were performed using the GraphPad Prism 5.1 program. Student T-test and Oneway ANOVA were used to calculate $\mathrm{p}$ value. Results are presented as mean \pm standard deviation.

\section{Results}

Sox2 suppresses the invasiveness of breast cancer cells Using an in-vitro assay, we assessed if Sox2 regulates the invasiveness of two ER + breast cancer cell lines (i.e., MCF7 and ZR751), both of which have shown the highest expression level of Sox2 described in our previous study [39]. As shown in Figure 1A, siRNA knockdown of Sox2 resulted in a significant increase in the invasiveness of MCF7 and ZR751 cells. These changes were not due to a difference in cell growth between cells treated with Sox2 siRNA or scramble siRNA (Figure 1B). In contrast with the findings of another group [38], we found no significant difference in the invasiveness between MCF7 cells transfected with an empty vector or a Sox2 expression vector (Figure 1C-D).

The suppression of invasiveness by Sox 2 is dependent on the status of the Sox 2 transcription activity

As Sox2 is a transcription factor, we asked if Sox2 is transcriptionally active in $\mathrm{BC}$ cells, and whether the status of its activity has any impact on its effect on the invasiveness in BC.

To assess the Sox2 transcriptional activity, we have employed a previously characterized Sox 2 reporter. The read-out of the reporter is provided by the inclusion of green fluorescence protein (GFP), driven by a $m C M V$ promoter [39]. With the Sox2 reporter employed, we had identified that MCF7 and ZR751 cells are composed of two phenotypically distinct cell subsets that can be separated based on their differential responsiveness to the Sox2 reporter [39]. Specifically, cells showing Sox2 transcriptional activity are GFP-positive whereas those showing no evidence of Sox2 transcriptional activity are GFP-negative [39]. For the purpose of this study, the former cell population is labeled 'reporter responsive' or RR cells and the latter cell population is labeled 'reporter 


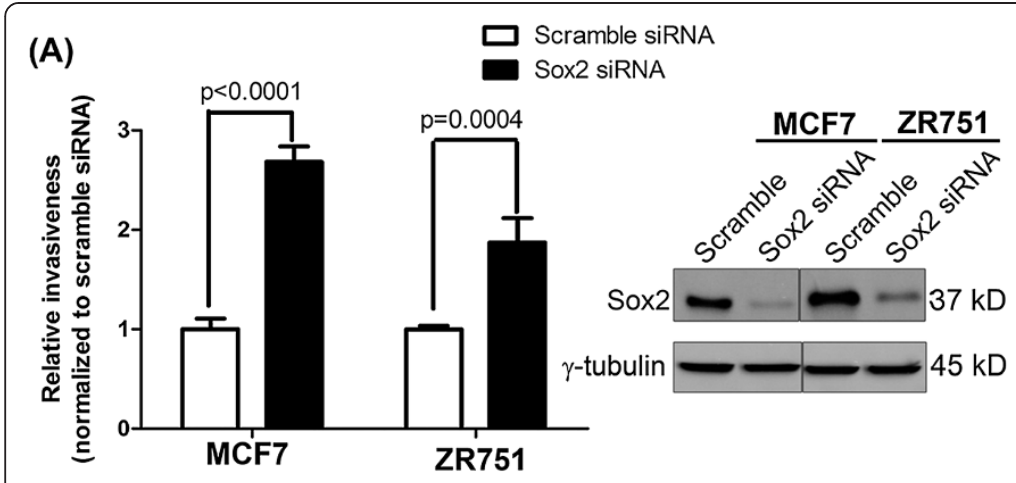

(B)

(C)
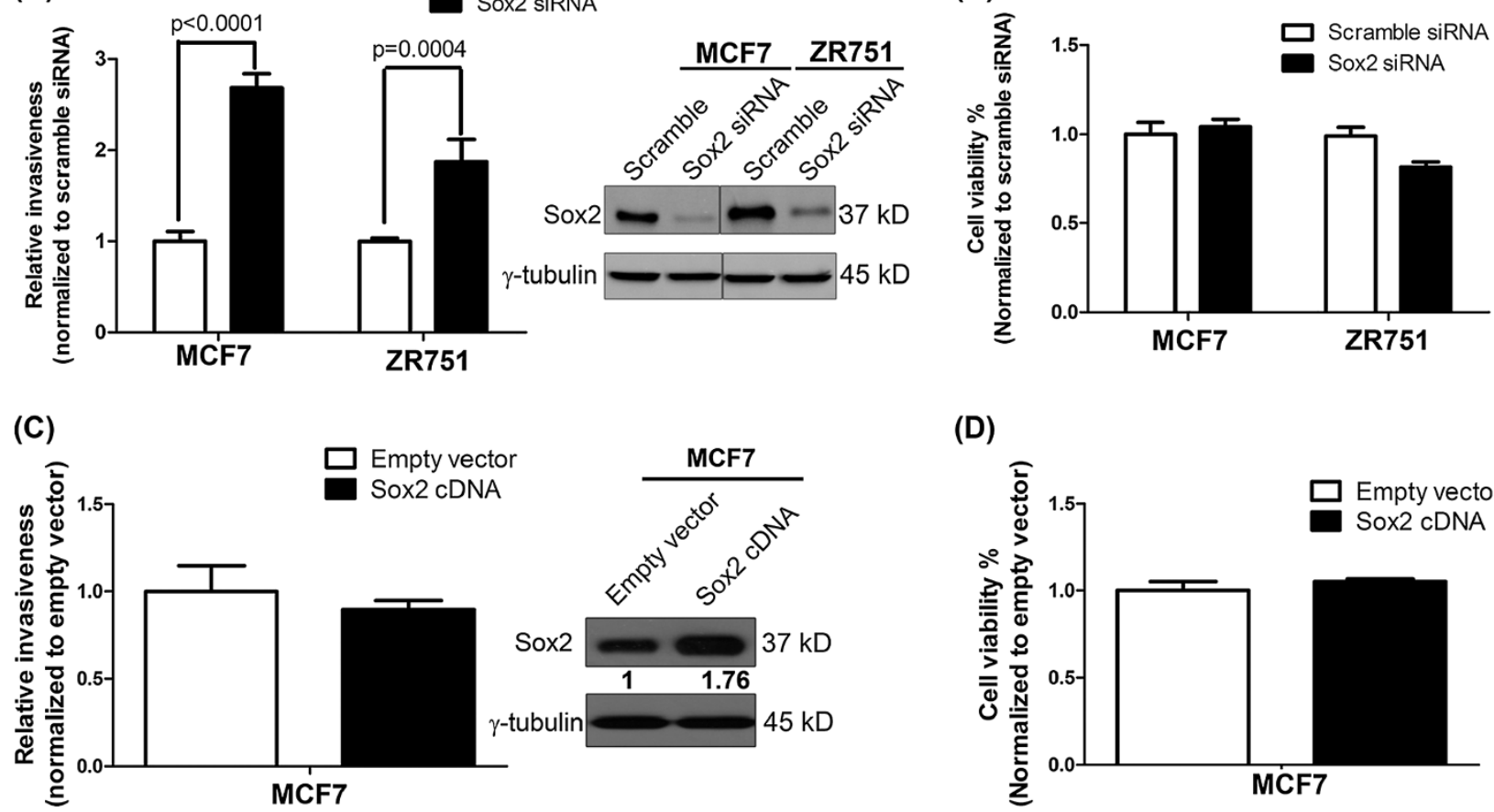

(D)

Figure 1 Sox2 suppresses invasiveness in breast cancer cells. (A) MCF7 and ZR751 cells were treated with Sox2 siRNA before subjecting to invasion assay. siRNA knockdown of Sox2 significantly increased the invasiveness of MCF7 and ZR751cells. A scrambled siRNA sequence was used as a control and results were normalized to the control. Triplicate experiments were performed. A representative experiment is shown (mean \pm standard deviation) $(n=3)$. Statistical significance was determined by Student's T-test. Western blots analysis showed that siRNA knockdown of Sox 2 dramatically decreased the expression of Sox2 in MCF7 and ZR751. (B) Cell viability was measured by the MTS assay. siRNA knockdown did not significantly change the viability of MCF7 cells. Similar results were obtained from ZR751 cells. Triplicate experiments were performed (mean \pm standard deviation) ( $\mathrm{n}=6$ ). Statistical significance was determined by Student's T-test. (C) Enforced expression of Sox2 did not significantly change the invasiveness of MCF7. MCF7 cells transfected with empty vector were used as a control. Triplicate experiments were performed. A representative experiment is shown (mean \pm standard deviation) $(n=3)$. (D) Cell viability was measured by the MTS assay. Enforced expression of Sox 2 did not significantly change the viability of MCF7 cells. Triplicate experiments were performed (mean \pm standard deviation) $(n=6)$.

un-responsive' or RU cells. To facilitate our studies, we generated stable cell clones expressing the Sox2 reporter construct. RR and RU cells were further isolated by flow cytometry and cultured separately. As shown in Additional file 1: Figure S1, the RR and RU cells were readily identified using flow cytometry. Cells stably transfected with the Sox 2 reporter that have not been sorted into RR and RU cells are labeled 'Sox2R'. We have previously excluded the possibility that the absence of GFP expression in RU cells is due to a lack of Sox 2 protein as the vast majority of MCF7 and ZR751 cells expressed Sox2 detected by flow cytometry. Furthermore, by subcellular fractionation, we confirmed that Sox 2 is present in the nuclei of these cells [39].

When the invasiveness of RR cells, RU cells and the unsorted Sox2R cells derived from MCF7 was compared, no significant difference was observed among these three cell populations (Figure 2A). However, as shown in Figure 2B, siRNA knockdown of Sox2 resulted in a significant increase in the invasiveness in MCF7-RU cells; in contrast, no significant change was seen in MCF7-RR cells. This difference between the two cell subsets was not due to a significant difference in their cell growth (Figure 2C). In keeping with our previous observation [39], siRNA knockdown of Sox2 also did not result in any significant change in the viability of MCF7-RR and RU cell populations (Figure 2D). The similar experiments were performed using ZR751-RU cells. In keeping with the results of MCF7 cells, siRNA knockdown of Sox2 in ZR751-RU cells significantly increased in the invasiveness (Figure 2E).

\section{Sox2 regulates Twist1 expression, but only in $\mathrm{RU}$ cells}

To understand the mechanism by which Sox 2 regulates the invasiveness of the RU cells, we examined if Sox2 modulates the expression of factors known to play key roles in regulating the invasiveness and/or EMT in various types of cancers, including Snail1, Slug, ZEB1, MMP2, MMP3, MMP9, Twist1, E-cadherin, N-cadherin, FAK and TGF- $\beta$ [43-47]. Using quantitative RT-PCR, we found that siRNA knockdown of Sox2 in both MCF7-RR and -RU cells did not result in significant changes in the 


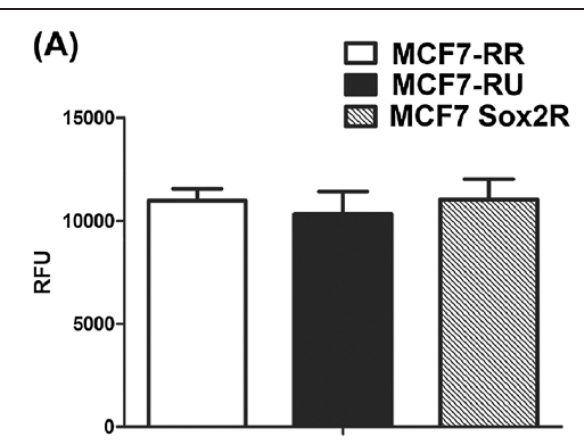

(C)

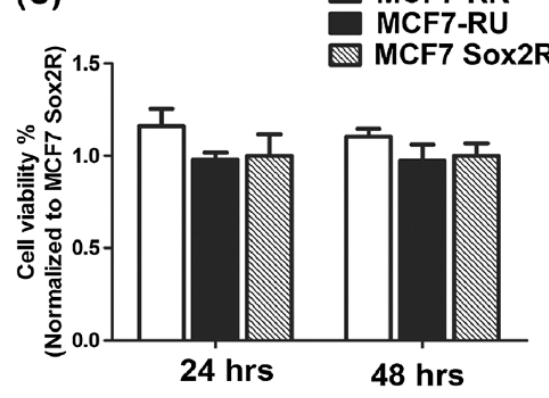

(E)

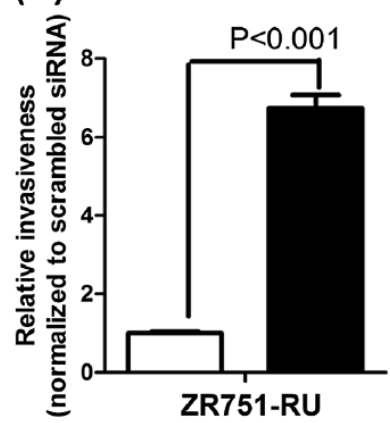

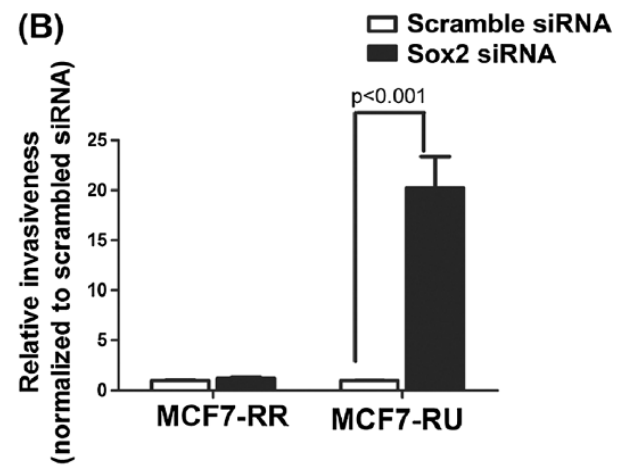

(D)

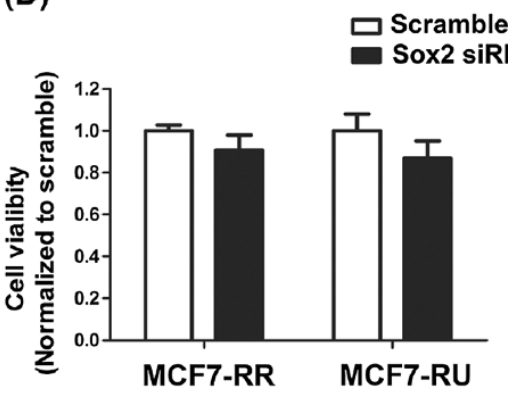

Figure 2 The suppressive effect of Sox 2 on the invasiveness in RU subset but not RR subset. (A) Cell invasiveness was also assessed using RR cells, RU cells and unsorted cells (labeled as 'Sox2R') derived from MCF7. No significant difference in invasiveness was observed between these three cell populations. Triplicate experiments were performed. A representative experiment is shown (mean \pm standard deviation) ( $n=3$ ). (B) MCF7-RR and -RU cells were subjected to either scramble siRNA or Sox2 siRNA treatment for 24 hour before invasion assay. Sox2 siRNA treatment resulted in significant increase in invasiveness in MCF7-RU cells; no significant change was observed in MCF7-RR cells. Triplicate experiments were performed. A representative experiment is shown (mean \pm standard deviation) $(n=3)$. (C) Cell viability of $R R$, $R U$, and unsorted cells (labeled as 'Sox2R) from MCF7 were assessed by the MTS assay. (D) MCF7-RR and -RU cells were treated with Sox2 siRNA or scramble siRNA before the MTS assay. No significant change in cell viability was found after Sox2 siRNA treatment. (E) ZR751-RU cells were subjected to either scramble siRNA or Sox2 siRNA treatment for 24 hour before invasion assay. Sox2 siRNA treatment significantly increases the invasiveness in ZR751-RU cells. Cell viability assay was also performed and no significant change was observed after Sox2 siRNA treatment.

mRNA levels of Snail1, Slug, ZEB1 (Figure 3B), as well as $M M P 2, M M P 3, M M P 9, F A K$ and TGF- $\beta$ (not shown). As shown in Figure $3 \mathrm{~B}$ and $\mathrm{C}$, we found that siRNA knockdown of Sox2 led to a significant up-regulation of the Twist1 mRNA as well as an upregulation of the Twist1 protein, although these changes were confined to the RU cells. Correlating with these findings, the expres- sion level of E-cadherin, one of the key Twist1 downstream targets, was down-regulated in RU cells but not RR cells (Figure 3B). N-cadherin, a cell-cell adhesion mediator, was significantly up-regulated in MCF7-RU cells but not -RR cells. Using ChIP assay, we were able to demonstrate that Sox 2 was bound to the promoter region of Twist 1 in RU cells but not RR cells (Figure 3D). 


\section{(A)}

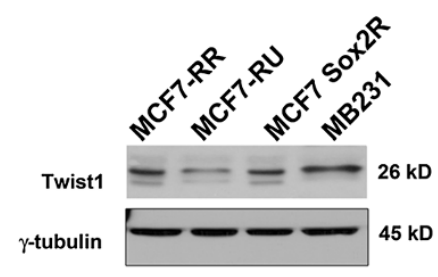

(B)
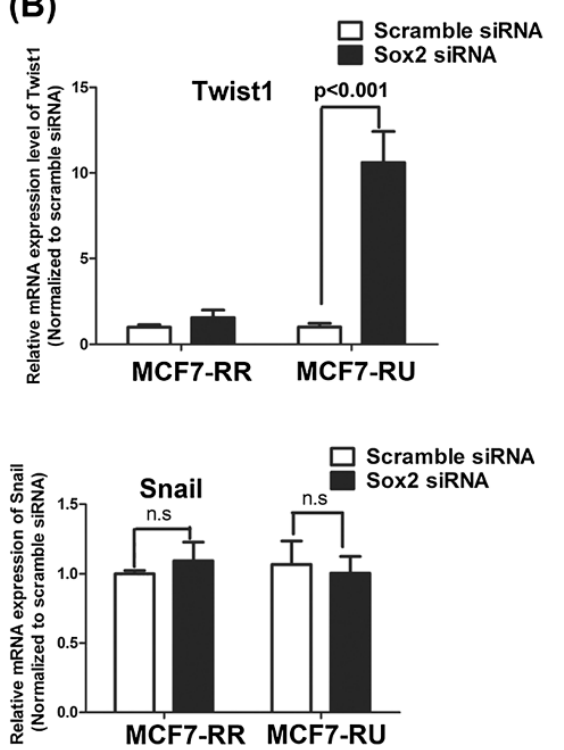

(C)

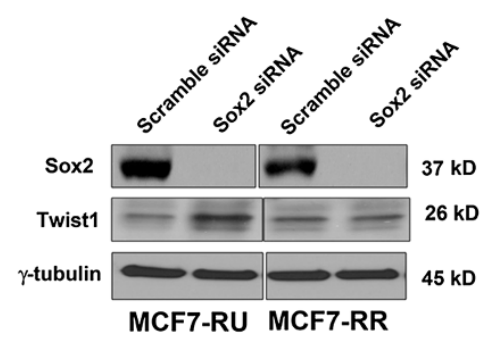

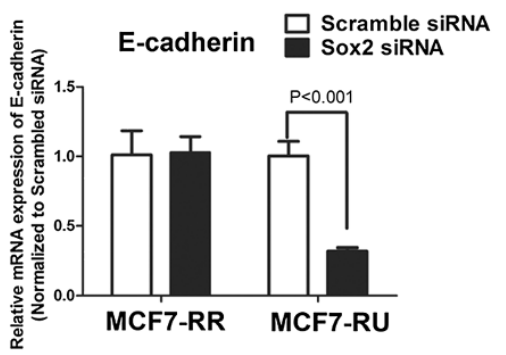
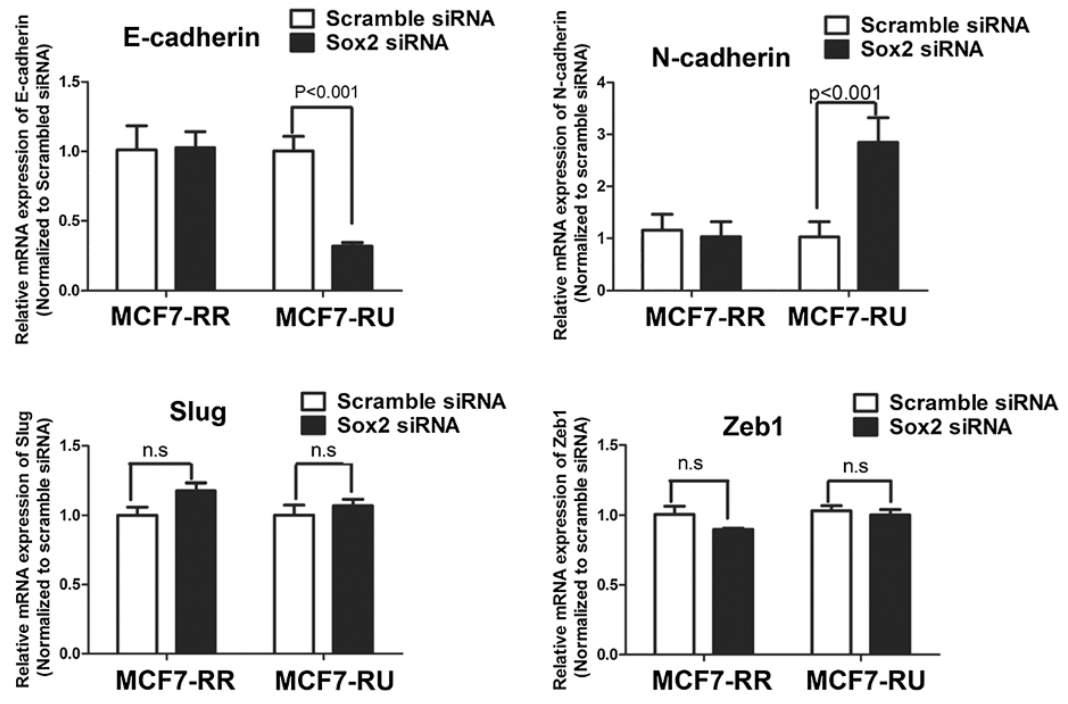

(D)

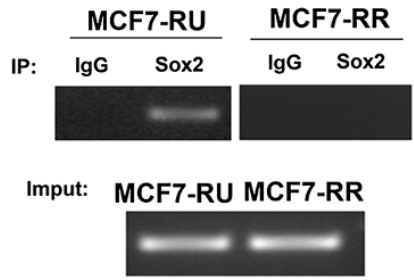

Figure 3 Modulation of Twist 1 expression by Sox 2 in RU cells but not RR cells. (A) By western blot analysis, the protein expression of Twist1 was examined in RR, RU and unsorted cells (labeled as 'Sox2R') from MCF7. MB231 was used as positive control. (B) MCF7-RR and -RU cells were treated with either scramble siRNA or Sox2 siRNA. By quantitative RT-PCR, the expression level of a panel of EMT/invasiveness inducers were examined, including Snail1, Slug, ZEB1, MMP2, MMP3, MMP9, Twist1, E-cadherin, N-cadherin, FAK and TGF- $\beta$. siRNA knockdown of Sox2 resulted in significant up-regulation of Twist1 and N-cadherin, down-regulation of E-cadherin in MCF7-RU cells but not -RR cells. No significant change was found in the expression level of Slug, Snail, and ZEB1, MMP2, MMP3, MMP9, FAK and TGF- $\beta$ after siRNA knockdown of Sox2. Three representative results (i.e., Slug, Snail, and ZEB1) were shown. Scramble siRNA was used as a control. (C) By western blot analysis, the protein expression level of Twist1 was detected after siRNA knockdown of Sox2. (D) For the ChIP assay, a normal rabbit lgG antibody or a specific anti-Sox2 antibody was incubated with cross-linked chromatin extracted from MCF7-RR and -RU cells. Isolated DNA was amplified with primer designed against the proximal promoter of Twist1. Sox2 was found to bind to the gene promoter region of Twist1 only in RU but not RR cells. Input control that represents DNA isolated from chromatin before immunoprecipitation shows equal loading. n.s represents no significant difference.

\section{Modulation of cell invasiveness by Sox 2 is mediated via Twist1}

We then asked if the Sox2-mediated modulation of invasiveness in RU cells is dependent on Twist1. As shown in Figure 4, siRNA knockdown of Sox2 in MCF7-RU cells led to a significant increase in invasiveness, whereas siRNA knockdown of Twist1 led to a significant decrease in invasiveness. Importantly, simultaneous silencing of Sox 2 and Twist 1 using siRNA largely abrogated the suppressive effect of Sox 2 on invasiveness in MCF7-RU cells. These findings strongly suggest that Sox 2 suppresses the invasiveness property of RU cells via down-regulating Twist1 in these cells. The same experiment was repeated using MCF7-RR cells and we found no significant change 


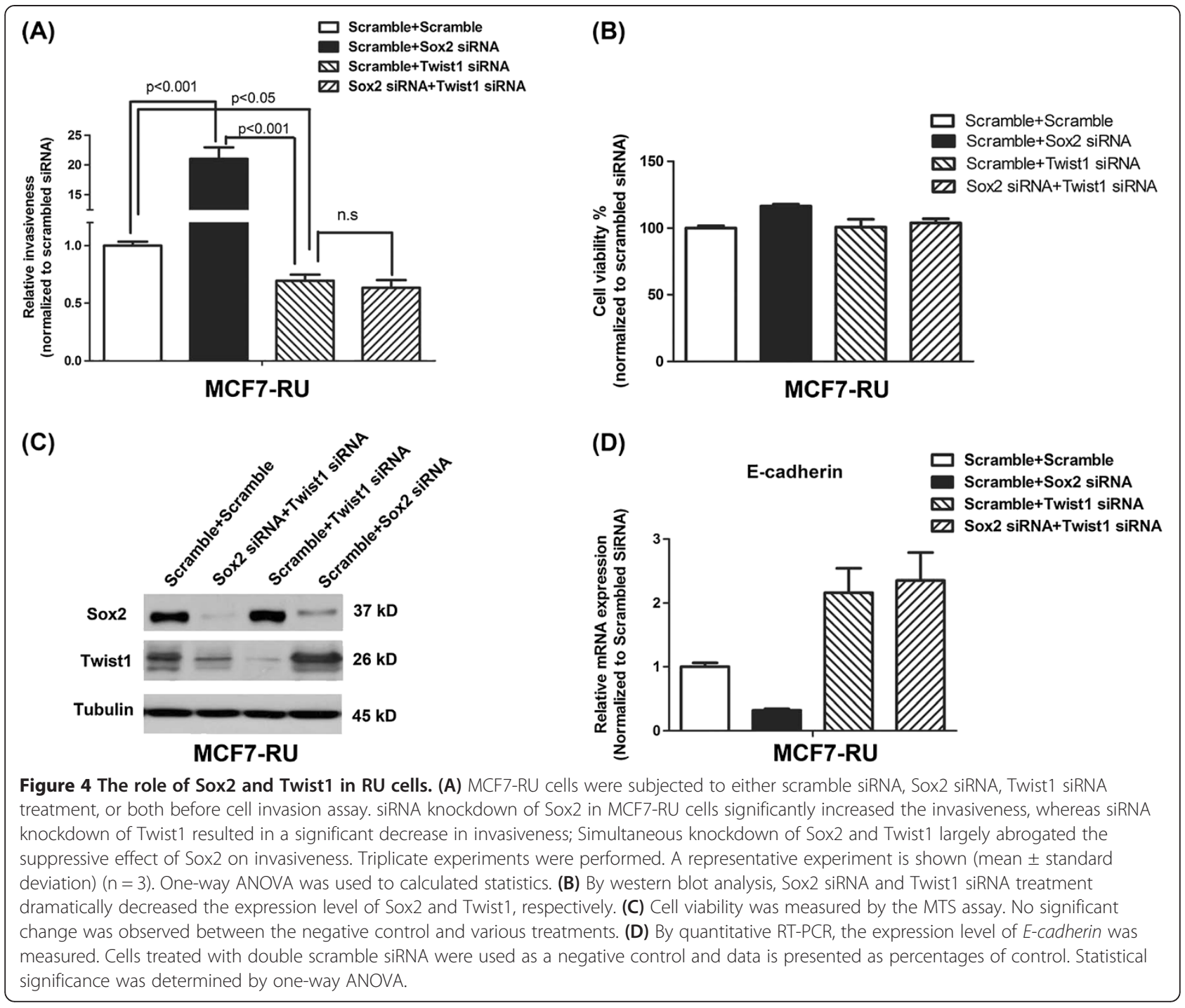

in the invasiveness of these cells (Figure 5). Nevertheless, siRNA knockdown of Twist1 resulted in a significant decrease in the invasiveness of MCF7-RR cells, suggesting that Twist1, but not Sox2, is a key regulator of invasiveness in these cells. Again, the observed differences in invasiveness were not due to a significant difference in the cell growth among the negative controls and various treatment groups (Figure 5B).

\section{Discussion}

The aberrant expression of Sox 2 in cancer cells has been found to correlate with the invasiveness of several types of solid tumors [30,34,35,37,48-50]. For instance, a high level of Sox 2 expression detectable by immunohistochemistry was found to correlate with higher invasiveness and metastatic potential in gliomas and colorectal cancer $[35,36]$. Furthermore, siRNA knockdown of Sox 2 can result in decreased invasiveness in cell lines derived from gliomas, melanomas and colorectal cancer [35-37]. However, it appears that Sox 2 expression in cancer does not always correlate with increased invasiveness and metastasis. We found at least one previous study in which a relatively low level of Sox 2 expression in gastric cancer correlates with increased invasiveness/metastatic potential [34]. In the current study, we also found evidence that Sox2 suppresses invasiveness in BC. Thus, the biological effects of Sox 2 in cancer cells are likely to be tumor type-specific.

Our finding that Sox 2 suppresses the invasiveness of $\mathrm{BC}$ is in contrast with that made by another group, who found that enforced expression of Sox 2 in MCF7 cells can increases their invasiveness by approximately $60 \%$ [38]. In our study, we initially found that siRNA knockdown of Sox2 significantly increased the invasiveness of parental MCF7 cells and MCF7-RU cells. In view of the discrepancy between our conclusion and that described 


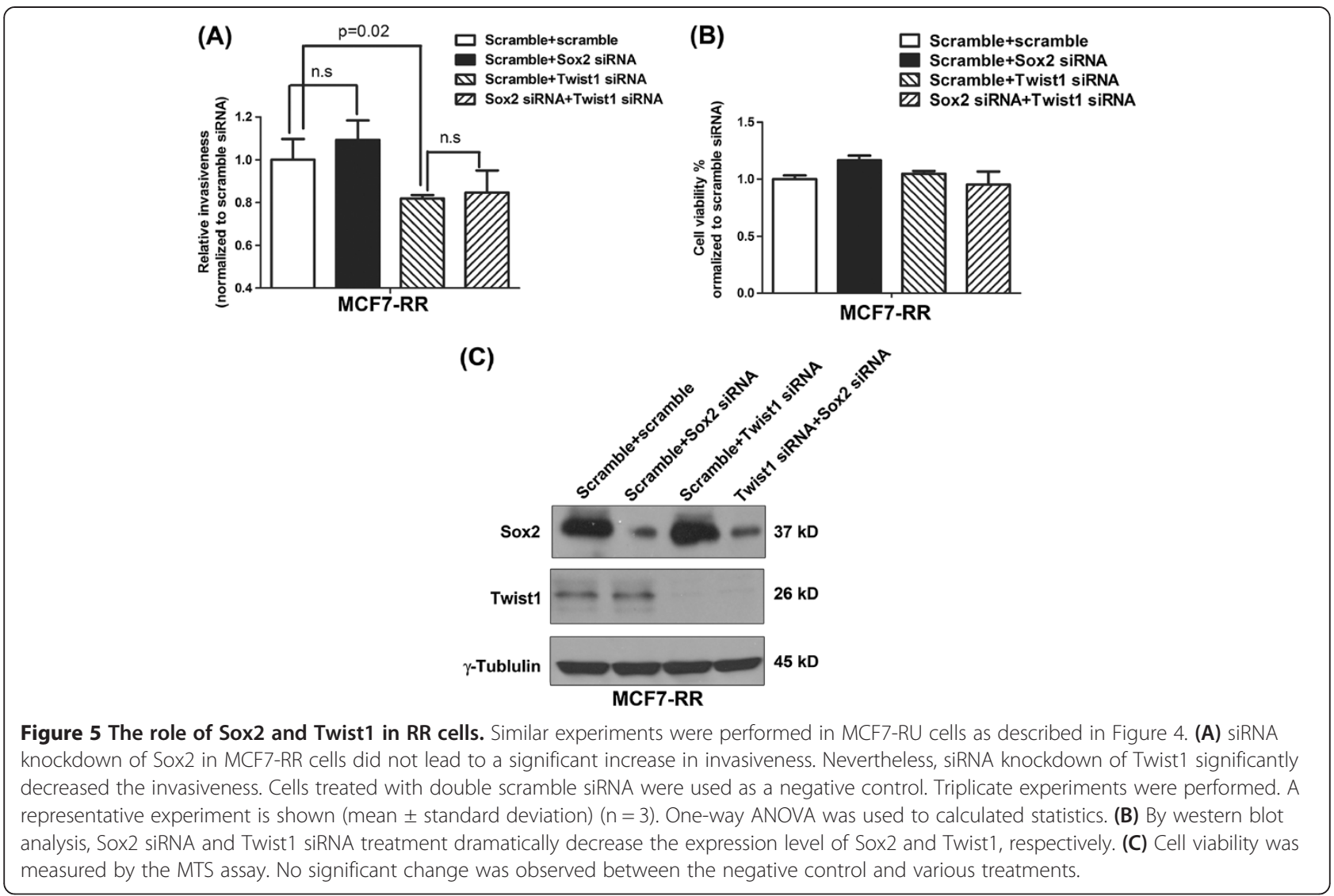

in the literature [38], we attempted to replicate the experiment that examined the effects of enforced Sox2 over-expression in MCF7 cells, as described previously [38], and we did not find any significant change in the invasiveness of these cells (Figure 1C). We would like to point out that the lack of response to enforced Sox2 expression in MCF7 is similar to the finding of one of previous studies, in which enforced expression of Sox 2 in MCF7 cells was found to result in no significant change in mammosphere formation and cell growth [39]. While we do not have definitive explanations for the discrepancy between our results and the previously published results [38], we have considered the possibility that the MCF7 cell clones used in the two laboratories may be different. We also have considered the possibility that the in-vitro invasiveness assays between the two laboratories have different characteristics. Lastly, since the exact Sox 2 protein level has been shown to be functionally important in ESCs [51,52], it is possible that the total Sox 2 protein levels after gene transfection are substantially different between the two laboratories, and thus, leading to substantially different biological responses.

The mechanisms by which Sox 2 regulate tumor invasiveness have not been extensively studied. In the literature, we were able to identify only 3 studies that are directly relevant to this subject. In all of these three studies (using cell lines derived from colorectal cancer, melanomas and gliomas, respectively), siRNA knockdown of Sox 2 was found to decrease invasiveness; in the same three studies, the decrease in invasiveness was found to correlate with a decreased expression level of one of the following molecules: MMP2, MMP3 or FAK $[36,37,53]$. To our knowledge, the mechanisms by which Sox2 regulates invasiveness in $\mathrm{BC}$ are not known. Thus, we screened a panel of factors known to play roles in regulating cell invasiveness/EMT in various types of cancer. In contrast with the previous reports, we did not find any appreciable changes in the expression levels of MMP3, MMP2 and FAK. Instead, we identified Twist1 as the only protein that is regulated by Sox 2 in RU cells.

Twist1 has been reported to be one of the master regulators of invasiveness and EMT, and dysregulation of Twist1 expression and function has been implicated to be associated with cancer progression [54-56]. In BC, a high level of Twist1 expression is more common in invasive lobular carcinomas [5]. While siRNA knockdown of Twist1 in BC cells led to a decrease in invasiveness [57], enforced expression of Twist1 in BC cells converts its normal epithelial cell morphology to a spindle-like/fibroblastic morphology [5,58]. In keeping with the concept that Twist1 plays a key role in regulating invasiveness in $\mathrm{BC}$, siRNA knockdown of Twist1 decreased the invasive- 
ness of both MCF7-RR and -RU cells by approximately 20$30 \%$ (Figures $4 \mathrm{~A}$ and $5 \mathrm{~A}$ ).

As mentioned in the introduction, the expression of Twist1 has been shown to be regulated by a number of proteins such as STAT3, BMP2 and SRC-1. The expression of Sox2 has been shown to correlate with that of Twist1 in human glioblastoma cells [59], although direct proof that Sox 2 regulates the expression of Twist1 is lacking. For the first time, we have provided direct evidence that the expression of Twist1 in BC is regulated by Sox2, and this regulation only occurs in the RU cells. Results from our ChIP studies further support the fact that Twist1 is regulated by Sox 2 only in RU cells. Although Sox 2 does not respond to the reporter in RU cells, possibly due to the fact that Sox2 in RU cells cannot bind to the Sox 2 binding motif present in the Sox2 reporter [39], Sox2 in RU cells can bind to the alternative Sox 2 binding motif present in the Twist1 gene promoter and thus suppress its expression as well as invasiveness. These findings are in parallel to the findings that Sox2 is known to negatively regulate a set of genes in ESCs. In contrast, in RR cells, Sox2 does not bind to the promoter region of Twist1 and the expression of Twist1 is regulated by other factors. The mechanism underlying the decision as to whether Sox 2 binds to the Twist1 gene promoter is under active investigation in our laboratory. Since the transcription activity of Sox 2 in normal ESCs has been shown to be modulated by its binding partners, we speculated that a similar scenario may occur in BC cells. Taken together, our findings suggest that the Sox2 transcriptional activity and Twist1 can serve as markers to predict invasiveness in breast cancer cells.

An important concept emerged from the results of this study is related to the significance of the dichotomy of BC cells separated based on the differential responsiveness to the Sox 2 reporter. Specifically, based on our double siRNA knockdown experiments (Figure 4), the Sox2-Twist1 axis plays a key role in regulating the invasiveness in RU cells. In contrast, Twist1, but not Sox2, plays a key role in regulating the invasiveness of $R R$ cells. While the true biological significance of these observations requires further studies, we believe that our results have highlighted a new level of biological complexity of $\mathrm{BC}$. In view of this new knowledge, one may wonder if our current treatments of $\mathrm{BC}$, which are designed based on the assumption that $\mathrm{BC}$ cells within a tumor are composed of a biologically uniform population of cancer cells, are fundamentally inadequate. This newly discovered biological complexity of BC cells may prompt us to consider treatment strategies that are based on the recognition of phenotypically distinct cell subsets in $\mathrm{BC}$ that are driven by different biochemical pathways.

\section{Conclusion}

In summary, we reported for the first time that Sox2 suppresses invasiveness in BC cells, but only in RU subset. Moreover, Sox 2 was found to be a major regulator of Twist1 by controlling the expression level of Twist1. Results from our studies have further supported that the dichotomy of $\mathrm{BC}$ based in their differential responsiveness to the Sox 2 reporter carries biological importance, highlighting a new level of biological complexity of $\mathrm{BC}$.

\section{Additional file}

Additional file 1: Figure S1. Identification of the dichotomy of BC cells based on the differential responsivenss to the Sox2 reporter. (A) MCF7 was stably transfected with either the Sox2 GFP reporter or mCMV lentiviral vector. Cells stably transfected with the Sox2 GFP reporter were labeled as 'MCF7 Sox2R'. Cells stably transfected with mCMV control were labeled as 'MCF7 mCMV'. GFP expression was measured by flow cytometry. Cells showing Sox2 transcriptional activity are GFP-positive whereas those showing no evidence of Sox2 transcriptional activity are GFP-negative. For the purpose of this study, the former cell population is labeled 'reporter responsive' or RR cells and the latter cell population is labeled 'reporter un-responsive' or RU cells. (B) To further examine the biology of these two cell subsets, we isolated and cultured the GFP-positive (labeled as 'RR') and GFP-negative cells (labeled as 'RU') separately from MCF7 cells.

\section{Abbreviations}

Sox2: Sex-determining region Y-box 2; GFP: Green fluorescent protein; ChIP: Chromatin immunoprecipitation; ESC: Embryonic stem cell; BC: Breast cancer; EMT: Epithelial-mesenchymal transition.

\section{Competing interests}

The authors declare that they have no competing interests.

\section{Authors' contributions}

FW and XY performed experiments and analyzed data; PW, KJ, CW, DD, NK, YM and GB assisted with experiments; FW and RL designed the research plan; FW and RL wrote the manuscript. All authors' read and approved the final manuscript.

\section{Acknowledgement}

This study was funded by the Canadian Institutes of Health Research and the Alberta Cancer Foundation awarded to R L. FW was awarded the Alberta Cancer Foundation Cancer Research Postdoctoral Fellowship. $\mathrm{KJ}$ is a recipient of the CIHR Vanier Canada Graduate Scholarship.

\section{Author details}

${ }^{1}$ Department of Laboratory Medicine and Pathology, University of Alberta, Edmonton, Alberta, Canada. ${ }^{2}$ Department of Oncology, University of Alberta, Edmonton, Alberta, Canada. ${ }^{3}$ Department of Surgery, University of Alberta, Edmonton, Alberta, Canada. ${ }^{4}$ Department of Pathology, Stonybrook University, Stonybrook, NY, USA. ${ }^{5}$ DynaLIFEDX Medical Laboratories, Edmonton, Alberta, Canada.

Received: 19 March 2013 Accepted: 19 June 2013

Published: 1 July 2013

\section{References}

1. Guarino M, Rubino B, Ballabio G: The role of epithelial-mesenchymal transition in cancer pathology. Pathology 2007, 39(3):305-318.

2. Thiery JP: Epithelial-mesenchymal transitions in tumour progression. Nat Rev Cancer 2002, 2(6):442-454.

3. Bastid J: EMT in carcinoma progression and dissemination: facts, unanswered questions, and clinical considerations. Cancer Metastasis Rev 2012, 31(1-2):277-283

4. Kalluri $R$, Weinberg RA: The basics of epithelial-mesenchymal transition. J Clin Invest 2009, 119(6):1420-1428. 
5. Yang J, Mani SA, Donaher JL, Ramaswamy S, Itzykson RA, Come C, Savagner P, Gitelman I, Richardson A, Weinberg RA: Twist, a master regulator of morphogenesis, plays an essential role in tumor metastasis. Cell 2004, 117(7):927-939.

6. Li QQ, Xu JD, Wang WJ, Cao XX, Chen Q, Tang F, Chen ZQ, Liu XP, Xu ZD: Twist1-mediated adriamycin-induced epithelial-mesenchymal transition relates to multidrug resistance and invasive potential in breast cancer cells. Clin Cancer Res 2009, 15(8):2657-2665.

7. Fu J, Qin L, He T, Qin J, Hong J, Wong J, Liao L, Xu J: The TWIST/Mi2/NuRD protein complex and its essential role in cancer metastasis. Cell Res 2011 , 21(2):275-289.

8. Cheng GZ, Chan J, Wang Q, Zhang W, Sun CD, Wang LH: Twist transcriptionally up-regulates AKT2 in breast cancer cells leading to increased migration, invasion, and resistance to paclitaxel. Cancer Res 2007, 67(5):1979-1987.

9. Vesuna F, Van Diest P, Chen JH, Raman V: Twist is a transcriptional repressor of E-cadherin gene expression in breast cancer. Biochem Biophys Res Commun 2008, 367(2):235-241.

10. Yang WH, Lan HY, Huang CH, Tai SK, Tzeng CH, Kao SY, Wu KJ, Hung MC, Yang MH: RAC1 activation mediates Twist1-induced cancer cell migration. Nat Cell Biol 2012, 14(4):366-374.

11. Tan EJ, Thuault S, Caja L, Carletti T, Heldin CH, Moustakas A: Regulation of transcription factor Twist expression by the DNA architectural protein high mobility group A2 during epithelial-to-mesenchymal transition. J Biol Chem 2012, 287(10):7134-7145.

12. Hong J, Zhou J, Fu J, He T, Qin J, Wang L, Liao L, Xu J: Phosphorylation of serine 68 of Twist1 by MAPKs stabilizes Twist 1 protein and promotes breast cancer cell invasiveness. Cancer Res 2011, 71(11):3980-3990.

13. Cheng GZ, Zhang WZ, Sun M, Wang Q, Coppola D, Mansour M, Xu LM, Costanzo C, Cheng JQ, Wang LH: Twist is transcriptionally induced by activation of STAT3 and mediates STAT3 oncogenic function. J Biol Chem 2008, 283(21):14665-14673.

14. Ma L, Lu MF, Schwartz RJ, Martin JF: Bmp2 is essential for cardiac cushion epithelial-mesenchymal transition and myocardial patterning Development 2005, 132(24):5601-5611.

15. Qin $L$, Liu Z, Chen $H_{1}, X u$ J: The steroid receptor coactivator-1 regulates twist expression and promotes breast cancer metastasis. Cancer Res 2009, 69(9):3819-3827.

16. Satoh K, Hamada S, Kimura K, Kanno A, Hirota M, Umino J, Fujibuchi W, Masamune A, Tanaka N, Miura K, et al: Up-regulation of MSX2 enhances the malignant phenotype and is associated with twist 1 expression in human pancreatic cancer cells. Am J Pathol 2008, 172(4):926-939.

17. Li CW, Xia W, Huo L, Lim SO, Wu Y, Hsu JL, Chao CH, Yamaguchi H, Yang NK, Ding Q, et al: Epithelial-mesenchymal transition induced by TNF-alpha requires NF-kappaB-mediated transcriptional upregulation of Twist1. Cancer Res 2012, 72(5):1290-1300

18. Kalra J, Sutherland BW, Stratford AL, Dragowska W, Gelmon KA, Dedhar S, Dunn SE, Bally MB: Suppression of Her2/neu expression through ILK inhibition is regulated by a pathway involving TWIST and YB-1. Oncogene 2010, 29(48):6343-6356.

19. Nairismagi ML, Vislovukh A, Meng Q, Kratassiouk G, Beldiman C, Petretich M, Groisman R, Fuchtbauer EM, Harel-Bellan A, Groisman I: Translational control of TWIST1 expression in MCF-10A cell lines recapitulating breast cancer progression. Oncogene 2012, 31(47):4960-4966.

20. Yang F, Sun L, Li Q, Han X, Lei L, Zhang H, Shang Y: SET8 promotes epithelial-mesenchymal transition and confers TWIST dual transcriptional activities. EMBO J 2012, 31(1):110-123.

21. Haque I, Banerjee S, Mehta S, De A, Majumder M, Mayo MS, Kambhampati S, Campbell DR, Banerjee SK: Cysteine-rich 61-connective tissue growth factor-nephroblastoma-overexpressed 5 (CCN5)/Wnt-1-induced signaling protein-2 (WISP-2) regulates microRNA-10b via hypoxia-inducible factor1alpha-TWIST signaling networks in human breast cancer cells. J Biol Chem 2011, 286(50):43475-43485.

22. Li S, Kendall SE, Raices R, Finlay J, Covarrubias M, Liu Z, Lowe G, Lin YH, Teh YH, Leigh V, et al: TWIST1 associates with NF-kappaB subunit RELA via carboxyl-terminal WR domain to promote cell autonomous invasion through IL8 production. BMC Bio/ 2012, 10:73.

23. Eckert MA, Lwin TM, Chang AT, Kim J, Danis E, Ohno-Machado L, Yang J: Twist1-induced invadopodia formation promotes tumor metastasis. Cancer Cell 2011, 19(3):372-386.
24. Keramari M, Razavi J, Ingman KA, Patsch C, Edenhofer F, Ward CM, Kimber SJ: Sox2 is essential for formation of trophectoderm in the preimplantation embryo. PLoS One 2010, 5(11):e13952.

25. Adachi K, Suemori H, Yasuda SY, Nakatsuji N, Kawase E: Role of SOX2 in maintaining pluripotency of human embryonic stem cells. Genes Cells 2010, 15(5):455-470.

26. Kiefer JC: Back to basics: Sox genes. Dev Dyn 2007, 236(8):2356-2366.

27. Okita K, Yamanaka S: Induction of pluripotency by defined factors. Exp Cell Re 2010, 316(16):2565-2570.

28. Park $I H$, Zhao $R$, West JA, Yabuuchi A, Huo H, Ince TA, Lerou PH, Lensch MW, Daley GQ: Reprogramming of human somatic cells to pluripotency with defined factors. Nature 2008, 451(7175):141-146.

29. Annovazzi L, Mellai M, Caldera V, Valente G, Schiffer D: SOX2 expression and amplification in gliomas and glioma cell lines. CANCER GENOMICS PROTEOMICS 2011, 8(3):139-147.

30. Jia X, Li X, Xu Y, Zhang S, Mou W, Liu Y, Lv D, Liu CH, Tan X, Xiang R, et al: SOX2 promotes tumorigenesis and increases the anti-apoptotic property of human prostate cancer cell. J Mol Cell Biol 2011, 3(4):230-238.

31. Chen $Y$, Shi L, Zhang L, Li R, Liang J, Yu W, Sun L, Yang X, Wang Y, Zhang $Y$, et al: The molecular mechanism governing the oncogenic potential of SOX2 in breast cancer. J Biol Chem 2008, 283(26):17969-17978.

32. Chen $S, X u$ Y, Chen Y, Li X, Mou W, Wang L, Liu Y, Reisfeld RA, Xiang R, LV $D$, et al: SOX2 gene regulates the transcriptional network of oncogenes and affects tumorigenesis of human lung cancer cells. PLoS One 2012, 7(5):e36326.

33. Gelebart P, Hegazy SA, Wang P, Bone KM, Anand M, Sharon D, Hitt M, Pearson JD, Ingham RJ, Ma Y, et al: Aberrant expression and biological significance of Sox2, an embryonic stem cell transcriptional factor, in ALK-positive anaplastic large cell lymphoma. Blood Cancer J 2012, 2:e82.

34. Zhang X, Yu H, Yang Y, Zhu R, Bai J, Peng Z, He Y, Chen L, Chen W, Fang D, et al: SOX2 in gastric carcinoma, but not Hath1, is related to patients' clinicopathological features and prognosis. J Gastrointest Surg 2010, 14 (8):1220-1226

35. Alonso MM, Diez-Valle R, Manterola L, Rubio A, Liu D, Cortes-Santiago N, Urquiza L, Jauregi P, Lopez De Munain A, Sampron N, et al: Genetic and epigenetic modifications of Sox 2 contribute to the invasive phenotype of malignant gliomas. PLoS One 2011, 6(11):e26740.

36. Han X, Fang $X$, Lou $X$, Hua D, Ding W, Foltz G, Hood L, Yuan Y, Lin B: Silencing SOX 2 induced mesenchymal-epithelial transition and its expression predicts liver and lymph node metastasis of CRC patients. PLoS One 2012, 7(8):e41335

37. Girouard SD, Laga AC, Mihm MC, Scolyer RA, Thompson JF, Zhan Q, Widlund HR, Lee CW, Murphy GF: SOX2 contributes to melanoma cell invasion. Lab Invest 2012, 92(3):362-370.

38. Simoes BM, Piva M, Iriondo O, Comaills V, Lopez-Ruiz JA, Zabalza I, Mieza JA, Acinas O, Vivanco MD: Effects of estrogen on the proportion of stem cells in the breast. Breast Cancer Res Treat 2011, 129(1):23-35.

39. Wu F, Zhang J, Wang P, Ye X, Jung K, Bone KM, Pearson JD, Ingham RJ, McMullen TP, Ma $Y$, et al: Identification of two novel phenotypically distinct breast cancer cell subsets based on Sox 2 transcription activity. Cell Signal 2012, 24(11):1989-1998.

40. Wu F, Wang P, Young LC, Lai R, Li L: Proteome-wide identification of novel binding partners to the oncogenic fusion gene protein, NPM-ALK, using tandem affinity purification and mass spectrometry. Am J Pathol 2009, 174(2):361-370.

41. Wu F, Wang P, Zhang J, Young LC, Lai R, Li L: Studies of phosphoproteomic changes induced by nucleophosmin-anaplastic lymphoma kinase (ALK) highlight deregulation of tumor necrosis factor (TNF)/Fas/TNF-related apoptosis-induced ligand signaling pathway in ALK-positive anaplastic large cell lymphoma. Mol Cell Proteomics 2010, 9(7):1616-1632.

42. Zhang J, Wang P, Wu F, Li M, Sharon D, Ingham RJ, Hitt M, McMullen TP, Lai R: Aberrant expression of the transcriptional factor Twist1 promotes invasiveness in ALK-positive anaplastic large cell lymphoma. Cell Signal 2012, 24(4):852-858.

43. Peinado $\mathrm{H}$, Olmeda D, Cano A: Snail, Zeb and bHLH factors in tumour progression: an alliance against the epithelial phenotype? Nat Rev Cancer 2007, 7(6):415-428.

44. Alkatout I, Wiedermann M, Bauer M, Wenners A, Jonat W, Klapper W: Transcription factors associated with epithelial-mesenchymal transition and cancer stem cells in the tumor centre and margin of invasive breast cancer. Exp Mol Pathol 2012. 
45. Barrallo-Gimeno A, Nieto MA: The Snail genes as inducers of cell movement and survival: implications in development and cancer. Development 2005, 132(14):3151-3161

46. Walsh LA, Damjanovski S: IGF-1 increases invasive potential of MCF 7 breast cancer cells and induces activation of latent TGF-beta1 resulting in epithelial to mesenchymal transition. Cell Commun Signal 2011, 9(1):10

47. Wang X, Lu H, Urvalek AM, Li T, Yu L, Lamar J, DiPersio CM, Feustel PJ, Zhao J: KLF8 promotes human breast cancer cell invasion and metastasis by transcriptional activation of MMP9. Oncogene 2011, 30(16):1901-1911.

48. Xiang R, Liao D, Cheng T, Zhou H, Shi Q, Chuang TS, Markowitz D, Reisfeld RA, Luo Y: Downregulation of transcription factor SOX2 in cancer stem cells suppresses growth and metastasis of lung cancer. Br J Cancer 2011, 104(9):1410-1417.

49. Matsuoka J, Yashiro M, Sakurai K, Kubo N, Tanaka H, Muguruma K, Sawada T, Ohira M, Hirakawa K: Role of the stemness factors sox2, oct3/4, and nanog in gastric carcinoma. J Surg Res 2012, 174(1):130-135.

50. Sanada Y, Yoshida K, Ohara M, Oeda M, Konishi K, Tsutani Y: Histopathologic evaluation of stepwise progression of pancreatic carcinoma with immunohistochemical analysis of gastric epithelial transcription factor SOX2: comparison of expression patterns between invasive components and cancerous or nonneoplastic intraductal components. Pancreas 2006, 32(2):164-170.

51. Kopp JL, Ormsbee BD, Desler M, Rizzino A: Small increases in the level of Sox2 trigger the differentiation of mouse embryonic stem cells. Stem Cells 2008, 26(4):903-911.

52. Chew JL, Loh YH, Zhang W, Chen X, Tam WL, Yeap LS, Li P, Ang YS, Lim B, Robson $P$, et al: Reciprocal transcriptional regulation of Pou5f1 and Sox2 via the Oct4/Sox2 complex in embryonic stem cells. Mol Cell Biol 2005, 25 (14):6031-6046.

53. Oppel F, Muller N, Schackert G, Hendruschk S, Martin D, Geiger KD, Temme A: SOX2-RNAi attenuates S-phase entry and induces RhoA-dependent switch to protease-independent amoeboid migration in human glioma cells. Mol Cancer 2011, 10:137.

54. Hoek K, Rimm DL, Williams KR, Zhao H, Ariyan S, Lin A, Kluger HM, Berger AJ, Cheng E, Trombetta ES, et al: Expression profiling reveals novel pathways in the transformation of melanocytes to melanomas. Cancer Res 2004, 64(15):5270-5282.

55. Zhang Z, Xie D, Li X, Wong YC, Xin D, Guan XY, Chua CW, Leung SC, Na Y, Wang $X$ : Significance of TWIST expression and its association with E-cadherin in bladder cancer. Hum Pathol 2007, 38(4):598-606.

56. Watson MA, Ylagan LR, Trinkaus KM, Gillanders WE, Naughton MJ, Weilbaecher KN, Fleming TP, Aft RL: Isolation and molecular profiling of bone marrow micrometastases identifies TWIST1 as a marker of early tumor relapse in breast cancer patients. Clin Cancer Res 2007, 13(17):5001-5009.

57. Banerjee A, Wu ZS, Qian P, Kang J, Pandey V, Liu DX, Zhu T, Lobie PE: ARTEMIN synergizes with TWIST1 to promote metastasis and poor survival outcome in patients with ER negative mammary carcinoma. Breast Cancer Res 2011, 13(6):R112.

58. Mironchik Y, Winnard PT Jr, Vesuna F, Kato Y, Wildes F, Pathak AP, Kominsky S, Artemov D, Bhujwalla Z, Van Diest $P$, et al: Twist overexpression induces in vivo angiogenesis and correlates with chromosomal instability in breast cancer. Cancer Res 2005, 65(23):10801-10809.

59. Velpula KK, Dasari VR, Tsung AJ, Dinh DH, Rao JS: Cord blood stem cells revert glioma stem cell EMT by down regulating transcriptional activation of Sox2 and Twist1. Oncotarget 2011, 2(12):1028-1042.

\section{Submit your next manuscript to BioMed Central and take full advantage of:}

- Convenient online submission

- Thorough peer review

- No space constraints or color figure charges

- Immediate publication on acceptance

- Inclusion in PubMed, CAS, Scopus and Google Scholar

- Research which is freely available for redistribution

Submit your manuscript at www.biomedcentral.com/submit
C Biomed Central 